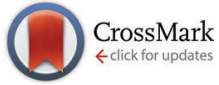

Cite this: Phys. Chem. Chem. Phys., 2016, 18, 10896

Received 22nd January 2016, Accepted 3rd March 2016

DOI: 10.1039/c6сp00487c

www.rsc.org/pccp

\section{The crystallization of metal soaps and fatty acids in oil paint model systems $\dagger$}

\begin{abstract}
Joen J. Hermans, ${ }^{* a}$ Katrien Keune, ${ }^{\text {ab }}$ Annelies van Loon ${ }^{a}$ and Piet D. ledema ${ }^{a}$
The formation and crystallization of metal soaps in oil paint layers is an important issue in the conservation of oil paintings. The chemical reactions and physical processes that are involved in releasing metal ions from pigments and fatty acids from the oil binder to form crystalline metal soap deposits have so far remained poorly understood. We have used a combination of differential scanning calorimetry (DSC) and attenuated total reflection Fourier transform infrared spectroscopy (ATR-FTIR) on model mixtures of palmitic acid, lead palmitate or zinc palmitate and linseed oil to study the transition from amorphous material to crystalline fatty acid or metal soap. This transition forms the final stage in the cascade of processes leading to metal soap-related oil paint degradation. Palmitic acid as well as the metal soaps showed nearly ideal solubility behavior. However, it was found that, near room temperature, both lead and zinc palmitate are practically insoluble in both liquid and partially polymerized linseed oil. Interestingly, the rate of metal soap and fatty acid crystallization decreased rapidly with the degree of linseed oil polymerization, possibly leading to systems where metal soaps are kinetically trapped in a semicrystalline state. To explain the various morphologies of metal soap aggregates observed in oil paint layers, it is proposed that factors affecting the probability of crystal nucleation and the rate of crystal growth play a crucial role, like exposure to heat or cleaning solvents and the presence of microcracks.
\end{abstract}

\section{Introduction}

In oil painting conservation, the formation of complexes of metal ions and saturated fatty acids, so-called metal soaps, is a growing concern. Analysis of small paint samples has revealed that large deposits of lead soaps ${ }^{1-4}$ and zinc soaps,${ }^{5-7}$ in some cases visible to the naked eye, have formed within paint layers or on the exposed surface of paintings ranging all the way from the 16th century to present times. Additionally, cases have been reported where saturated fatty acids have diffused out of a paint and crystallized on the paint surface. ${ }^{8-10}$ All these phenomena have a destructive effect not only on the appearance of an oil painting, but also on its structural integrity, since the formation of metal soaps is initiated by (partial) degradation of pigments and the binding medium. It is crucial for the preservation of oil paintings to know how to safely display, clean and restore these invaluable works of art without triggering metal soap related

\footnotetext{
${ }^{a}$ Van't Hoff Institute for Molecular Sciences, University of Amsterdam, PO box 94157, 1090GD Amsterdam, The Netherlands. E-mail: j.j.hermans@uva.nl; Tel: +31 (0)20 5256442

${ }^{b}$ Rijksmuseum Amsterdam, Conservation and Restoration, PO box 74888 , 1070DN Amsterdam, The Netherlands

$\dagger$ Electronic supplementary information (ESI) available: Details of DSC programs; heating rate dependence of melting/crystallization temperature hysteresis; illustrations of the crystalline structures of $\mathrm{HPa}, \mathrm{PbPa}$ and $\mathrm{ZnPa}$; temperaturedependent ATR-FTIR spectra of PbPa and ZnPa. See DOI: 10.1039/c6cp00487c
}

degradation phenomena or enhancing those phenomena that have already occurred.

In order to understand why metal soaps separate from the oil paint medium once they are formed and to explain why the metal soaps accumulate in such large aggregates, we use a combination of differential scanning calorimetry (DSC) and attenuated total reflection Fourier transform infrared spectroscopy (ATR-FTIR) to study the phase behavior of lead palmitate $(\mathrm{PbPa})$, zinc palmitate (ZnPa) and palmitic acid ( $\mathrm{HPa})$ in (polymerized) linseed oil. The thermal behavior of pure metal soaps and fatty acids has been the topic of numerous publications. ${ }^{11-16}$ For the study of the crystallization processes of metal soaps in oil paintings, we are interested in how the known phase transitions are affected by the presence of the surrounding linseed oil medium and the properties of this oil medium that change during polymerization and ageing. Ultimately, we aim to provide information on the solubility of metal soaps in oil paints and give insight into the kinetics of the processes that lead to metal soap and fatty acid crystallization.

Outside the field of art conservation, metal soaps find application as plasticizers and reinforcers in ionomeric polymers, ${ }^{17-19}$ and they are formed as a curing agent in vulcanizing rubber. ${ }^{20,21}$ They also serve as promising anti-corrosion coatings on metal surfaces $^{22-24}$ and are frequently used as precursors in the synthesis of nanoparticles and nanofilms. ${ }^{25}$ In all these cases, understanding the effect of the polymer or solution environment 
on the phase behavior and crystallization kinetics of metal soaps is highly relevant.

Lead and zinc soaps of palmitic or stearic acid are by far the most common metal soaps found in oil paintings. Pure palmitic acid is included in this study both because saturated fatty acid deposits are occasionally encountered on painting surfaces and because the availability of 'free' fatty acids (i.e. not covalently bound to the polymerized oil network) is thought to play a crucial role in the formation of metal soaps. ${ }^{26}$

In oil paintings, there is great variation in the molecular composition and structure of the polymerized linseed oil medium as a result of oil pre-treatment procedures, environmental conditions, and the presence of different metal-containing driers or pigments. ${ }^{27-29}$ As a start, we study model mixtures of palmitic acid, lead palmitate or zinc palmitate and either liquid or (partially) polymerized linseed oil. It is important to discuss the relation between these model mixtures and actual oil paintings. In oil paints, metal soaps form after reactions between inorganic pigments (often $2 \mathrm{PbCO}_{3} \cdot \mathrm{Pb}(\mathrm{OH})_{2}, \mathrm{~Pb}_{3} \mathrm{O}_{4}, \mathrm{~Pb}_{2} \mathrm{SnO}_{4}$ or $\mathrm{ZnO}$ ) and the linseed oil binding medium, either during paint drying or in fully polymerized paint layers. We have chosen mixtures of pre-made pure metal soaps and linseed oil as model systems. By doing do, we assume that in the cascade of processes that occur between the preparation of the paint and the appearance of metal soap crystalline phases, there is a point where dispersed amorphous metal soap molecules are formed. In other words, this assumption implies that the crystalline metal soaps that are observed in oil paint layers are not formed directly in some reaction between the pigment and saturated fatty acids on the pigment/medium interface. This choice of model system is supported by the observation that metal ions from pigments find their way into the polymerized oil network by association with carboxylate side chains, and that crystalline metal soaps are easily formed by exposing the metal ion-containing linseed oil to free fatty acids. ${ }^{26,30}$ The DSC measurements that are reported here are therefore meant to shed light on the final stages of the metal soap formation process, namely the transition from these amorphous species to crystalline metal soap phases. To generate amorphous metal soap species in our model systems, it is necessary to heat the systems to a homogeneous melt and study the crystallization process upon cooling. It is at present still unclear precisely what structure remains in molten metal soaps, though it is clear from the infrared spectra of molten metal soaps that all order is lost in the alkyl chains of the fatty acids while metal-carboxylate bonds persist. ${ }^{12,14,31}$ A final assumption is that the amorphous state in molten metal soaps is structurally similar to the intermediate metal soap phase that is formed in oil paint systems before crystallization.

\section{Experimental}

\subsection{Materials}

Cold-pressed untreated linseed oil (LO, Kremer Pigmente) and palmitic acid (HPa, 98\%, Acros) were used as received. Lead palmitate $(\mathrm{PbPa})$ and zinc palmitate $(\mathrm{ZnPa})$ were prepared by mixing aqueous solutions of lead or zinc nitrate $(>99.0 \%$, Sigma Aldrich) with an aqueous solution of palmitic acid containing triethylamine as a base. The procedure is described in detail in ref. 32. The purity of all compounds was confirmed by FTIR spectroscopy and powder X-ray diffraction.

\subsection{Methods}

Differential scanning calorimetry (DSC) traces for mixtures of $\mathrm{PbPa}$ and $\mathrm{ZnPa}$ in linseed oil were recorded on a Netzsch STA 449 F3 Jupiter in open $\mathrm{Al}_{2} \mathrm{O}_{3}$ cups in an argon atmosphere. For DSC traces of $\mathrm{HPa}$ in linseed oil, and for measurements of mixtures as a function of polymerization time, a Perkin Elmer Jade DSC coupled to a $-20{ }^{\circ} \mathrm{C}$ ethanol cooling pump was used. For these measurements, samples were sealed in aluminium pans and measured in a nitrogen atmosphere.

For all measurements of $\mathrm{HPa}, \mathrm{PbPa}$ and $\mathrm{ZnPa}$ in $\mathrm{LO}$, the mixtures were briefly heated with a heat gun above the melting point of the solid and allowed to cool back down to room temperature, to ensure an entirely even solid/liquid mixture before starting a DSC run. Details of the temperature programs employed during DSC measurements can be found in the ESI. $\dagger$

For measurements of mixtures as a function of polymerization time, portions of each mixture were weighed into open aluminium DSC pans, and placed into an air-circulated oven at $150{ }^{\circ} \mathrm{C}$. Every 30 minutes up to 3 hours, a pan of each mixture was removed from the oven, cooled to room temperature and sealed immediately afterwards. The sample weight after heat treatment was used for enthalpy calculations, though the sample weight loss during polymerization was never more than $2 \%$.

The reported transition temperatures correspond to peak maxima. Enthalpy values were calculated by taking the average of two separate integrations, representing the lower and upper estimate of the width of the base of the peak with a linear baseline. Error bars on experimental enthalpy values correspond to these estimated bounds of the integrated peak area.

Attenuated total reflection Fourier transform infrared spectra (ATR-FTIR) were recorded on a Varian 660-IR FT-IR spectrometer at $4 \mathrm{~cm}^{-1}$ resolution and averaged over 16 scans.

\section{Results}

\subsection{Palmitic acid in linseed oil}

Mixtures of palmitic acid and linseed oil were subjected to repeated heating and cooling cycles (HPa-LO, Fig. 1). The second heating curve of HPa-LO was chosen for the determination of melting transitions, because the first heating run proved to be poorly reproducible. The endotherms show a single melting peak for all concentrations of HPa. However, during cooling some peak shoulders or minor additional peaks were occasionally encountered, though these additional phenomena showed no consistent behavior. In the literature, pure palmitic acid is known to exhibit just a single liquid-solid transition upon heating. ${ }^{16}$ For the purposes of this paper, we have consistently taken the maximum of the main peak as the crystallization temperature and the sum of all peaks as the total crystallization enthalpy. 

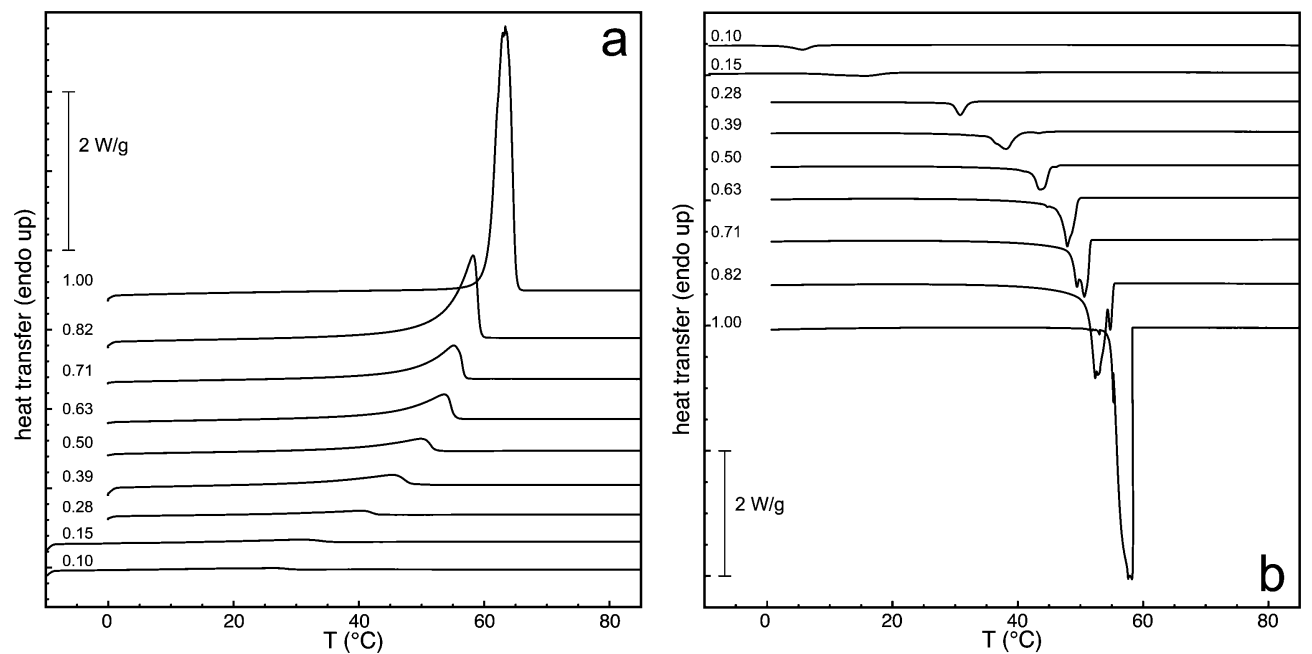

Fig. 1 (a) DSC second heating and (b) first cooling traces of HPa in linseed oil (2.5 $\mathrm{K} \mathrm{min}^{-1}$ ). Numbers indicate the molar fraction of HPa.

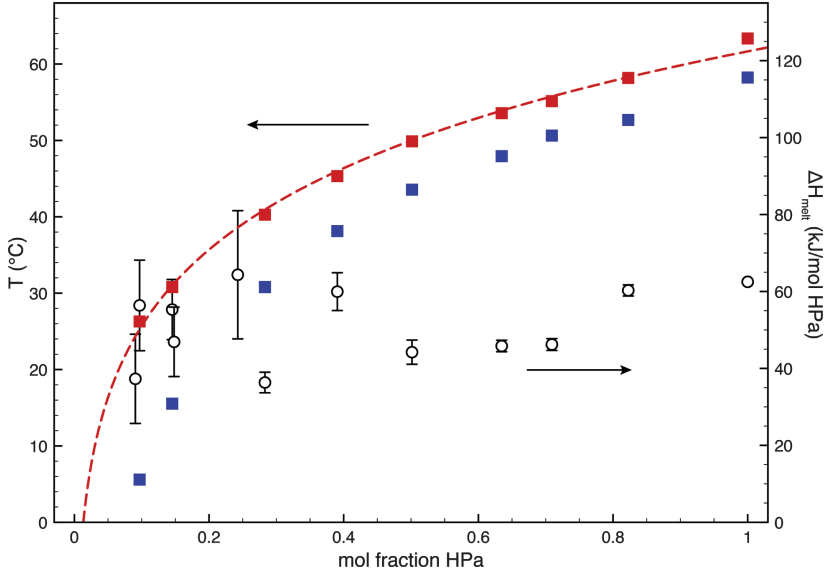

Fig. 2 Phase diagram of $\mathrm{HPa}$ in linseed oil with melting (red squares) and crystallization (blue squares) temperatures. The red dashed curve represents a fit of eqn (1). The corresponding melting enthalpy (circles) is indicated on the right axis.

Fig. 2 shows the melting and crystallization temperatures of the HPa-LO system, yielding a (partial) phase diagram of HPa as a function of concentration. The melting point of pure HPa at $63.4{ }^{\circ} \mathrm{C}$ is in the same range as the literature values collected by Cedeño and co-workers. ${ }^{16}$ As the concentration of $\mathrm{HPa}$ decreases, the melting temperature falls significantly. The corresponding enthalpy of melting, expressed per mol of $\mathrm{HPa}$ in the mixture, shows no clear dependence on HPa concentration. It is not known why the enthalpy values showed such a large spread and irreproducibility, indicated by the duplicate measurements at low HPa concentration in Fig. 2. Attempts to improve the reproducibility of the peak integrals by ensuring consistent sample pretreatment proved unsuccessful. The average value $\Delta H_{\text {melt }}$ of $52 \pm 9 \mathrm{~kJ} \mathrm{~mol}^{-1}$ is reasonably close to literature values. ${ }^{16,33}$

For ideal solutions, ${ }^{34}$ the saturation concentration of a solute A, expressed as molar fraction $x_{\mathrm{A}}$, is a function of temperature and is related to the melting temperature of the pure solid $T_{\text {melt }}$ and the corresponding melting enthalpy $\Delta^{\circ} H_{\text {melt }}$ according to

$$
\ln x_{\mathrm{A}}=\frac{\Delta^{\circ} H_{\text {melt }}}{R}\left(\frac{1}{T_{\text {melt }}}-\frac{1}{T}\right),
$$

in which $R$ is the ideal gas constant. This relation does not contain any solvent-solute interactions and it is therefore a description of ideal solubility behavior. When plotting $\ln x_{\mathrm{A}}$ as a function of measured values of $1 / T$, a linear correlation was found with slope $\Delta^{\circ} H_{\text {melt }} / R$ and intercept $\Delta^{\circ} H_{\text {melt }} / R T_{\text {melt }}$. Fig. 2 shows that eqn (1) fits the melting points measured in the HPa-LO system excellently. The obtained melting temperature of $61.7 \pm 0.8{ }^{\circ} \mathrm{C}$ and melting enthalpy of $53.3 \pm 1.3 \mathrm{~kJ} \mathrm{~mol}^{-1}$ are very close to the values reported by Cedeño and co-workers (see Table 1). ${ }^{16}$ This finding confirms the validity of eqn (1) and indicates nearly ideal behavior, implying only limited interaction between dissolved HPa and LO molecules. The topic of solution interactions is discussed further in terms of mixing enthalpy in Section 3.4.

Treating the fitted ideal solution curve as the phase boundary in the HPa-LO system, extrapolation yields a solubility of HPa in LO at $20{ }^{\circ} \mathrm{C}$ of approximately $6.6 \mathrm{~mol} \%$. The solubility of HPa as well as other thermodynamic data is compared with $\mathrm{PbPa}$ and ZnPa in Table 1.

The crystallization temperature of pure HPa was lower than the melting temperature by $5.2{ }^{\circ} \mathrm{C}$, and this hysteresis grew steadily to $20.7{ }^{\circ} \mathrm{C}$ for $10 \mathrm{~mol} \% \mathrm{HPa}$ in LO (Fig. 2). The observation of such

Table 1 Thermodynamic data obtained from DSC measurements of pure $\mathrm{HPa}, \mathrm{PbPa}$ and $\mathrm{ZnPa}$, and results of fits to eqn (1) (uncertainties in $T_{\text {melt }}$ and $\Delta^{\circ} H_{\text {melt }}$ from fits represent one standard deviation). The reported estimated solubility is at $20^{\circ} \mathrm{C}$

\begin{tabular}{lllllll}
\hline & $\begin{array}{l}T_{\text {melt }} \\
{\left[{ }^{\circ} \mathrm{C}\right]}\end{array}$ & $\begin{array}{l}T_{\text {crys }} \\
{\left[{ }^{\circ} \mathrm{C}\right]}\end{array}$ & $\begin{array}{l}\Delta^{\circ} H_{\text {melt }} \\
{\left[\mathrm{kJ} \mathrm{mol}^{-1}\right]}\end{array}$ & $\begin{array}{l}T_{\text {melt }} \\
\text { (fit) }\left[{ }^{\circ} \mathrm{C}\right]\end{array}$ & $\begin{array}{l}\Delta^{\circ} H_{\text {melt }} \text { (fit) } \\
{\left[\mathrm{kJ} \mathrm{mol}^{-1}\right]}\end{array}$ & $\begin{array}{l}\text { Solubility } \\
\text { (fit) }[\mathrm{mol} \%]\end{array}$ \\
\hline $\mathrm{HPa}$ & 63.4 & 58.2 & $62.5 \pm 0.6$ & $61.7 \pm 0.8$ & $53.3 \pm 1.3$ & 6.6 \\
$\mathrm{PbPa}$ & 114.3 & 110.0 & $103 \pm 3$ & $115 \pm 3$ & $98 \pm 4$ & $5 \times 10^{-3}$ \\
$\mathrm{ZnPa}$ & 131.8 & 89.9 & $85.6 \pm 0.2$ & $132 \pm 4$ & $106 \pm 5$ & $4 \times 10^{-4}$
\end{tabular}


temperature hysteresis indicates that a certain degree of supercooling is needed for the formation of critical crystallization nuclei. As such, hysteresis can be used as a measure of the rate of $\mathrm{HPa}$ crystallization. ${ }^{35}$ In the HPa-LO system, nucleation and/or growth of HPa crystalline phases became progressively slower with lower concentration (see also Fig. 4 and 6 for comparison). It was also found that temperature hysteresis varied linearly with heating rate for pure HPa and HPa-LO mixtures, while melting and crystallization enthalpy remained constant (see Fig. S1, ESI $\dagger$ ). These findings are consistent with the interpretation of temperature hysteresis as a measure of crystallization rate.

\subsection{PbPa in linseed oil}

Fig. 3 shows the endothermic and exothermic processes in the lead palmitate/linseed oil system (PbPa-LO). Pure PbPa shows two transitions upon heating: a transition to an intermediate solid rotator phase at $108.7^{\circ} \mathrm{C}$, and a transition to an isotropic liquid at $114.9^{\circ} \mathrm{C}^{12,14}$ When increasing quantities of linseed oil are mixed with $\mathrm{PbPa}$, the two transition temperatures move closer together and finally merge into one transition. A similar effect is seen in the cooling traces in Fig. 3, though the two distinct transitions persist until lower $\mathrm{PbPa}$ concentrations during crystallization.

The melting and crystallization temperatures of $\mathrm{PbPa}$ in oil as a function of concentration are shown in Fig. 4. The phase boundary shifts to lower temperatures with decreasing concentration, especially below $20 \mathrm{~mol} \% \mathrm{PbPa}$. For the determination of transition enthalpy in measurements that showed two distinct transitions, the two corresponding enthalpy values were summed to yield a total enthalpy for the transition from crystalline solid to isotropic liquid to allow a comparison of melting enthalpy over the entire $\mathrm{PbPa}$ concentration range, as shown in Fig. 4. The total enthalpy for the melting of pure $\mathrm{PbPa}$ is $103 \pm 3 \mathrm{~kJ} \mathrm{~mol}^{-1}$, which is in the same range as typical values encountered in the literature for lead palmitate $\left(118 \mathrm{~kJ} \mathrm{~mol}^{-112}\right.$ and $\left.96.4 \mathrm{~kJ} \mathrm{~mol}^{-111}\right)$. For the melting processes, expressed in terms of energy per mol of $\mathrm{PbPa}$ in the mixture, there is a clear trend towards lower values for lower

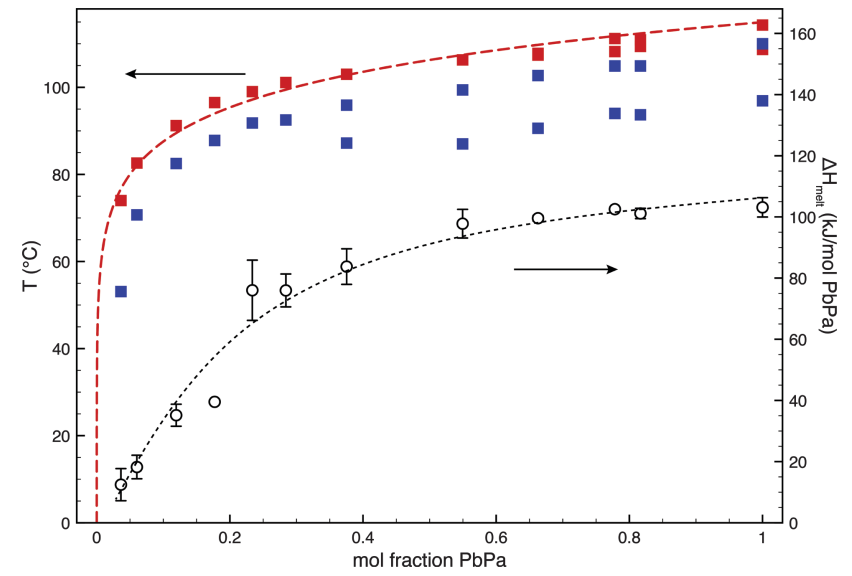

Fig. 4 Phase diagram of $\mathrm{PbPa}$ in linseed oil with melting (red squares) and crystallization (blue squares) temperatures. The red dashed curve represents a fit of eqn (1). The pair-wise occurrence of points at higher mol fractions corresponds to the twin peaks observed in Fig. 3, and indicates the presence of an intermediate solid rotator phase. The melting enthalpy (circles) is indicated on the left axis (dotted curve added for clarity).

$\mathrm{PbPa}$ concentrations, decreasing to only $18 \pm 4 \mathrm{~kJ} \mathrm{~mol}^{-1}$ at a $\mathrm{PbPa}$ mol fraction of 0.06 .

Again, we fitted the ideal solution phase boundary as described by eqn (1) to the melting temperatures of $\mathrm{PbPa}$ (Fig. 4). Similar to the HPa-LO system, the fit yields a melting temperature and melting enthalpy of pure $\mathrm{PbPa}$ close to the experimental values (Table 1). Using the ideal solution curve, the solubility of $\mathrm{PbPa}$ at $20{ }^{\circ} \mathrm{C}$ is estimated at only $5 \times 10^{-3} \mathrm{~mol} \%$.

In the $\mathrm{PbPa}-\mathrm{LO}$ system, temperature hysteresis is $6-9{ }^{\circ} \mathrm{C}$ and increases significantly only at very low concentrations, suggesting that the nucleation and growth of $\mathrm{PbPa}$ crystalline phases is a relatively fast process (see also Fig. 6 for comparison). As in the HPa-LO system, hysteresis was linearly dependent on heating rate while the melting enthalpy remained constant (see Fig. S1, ESI $\dagger$ ).
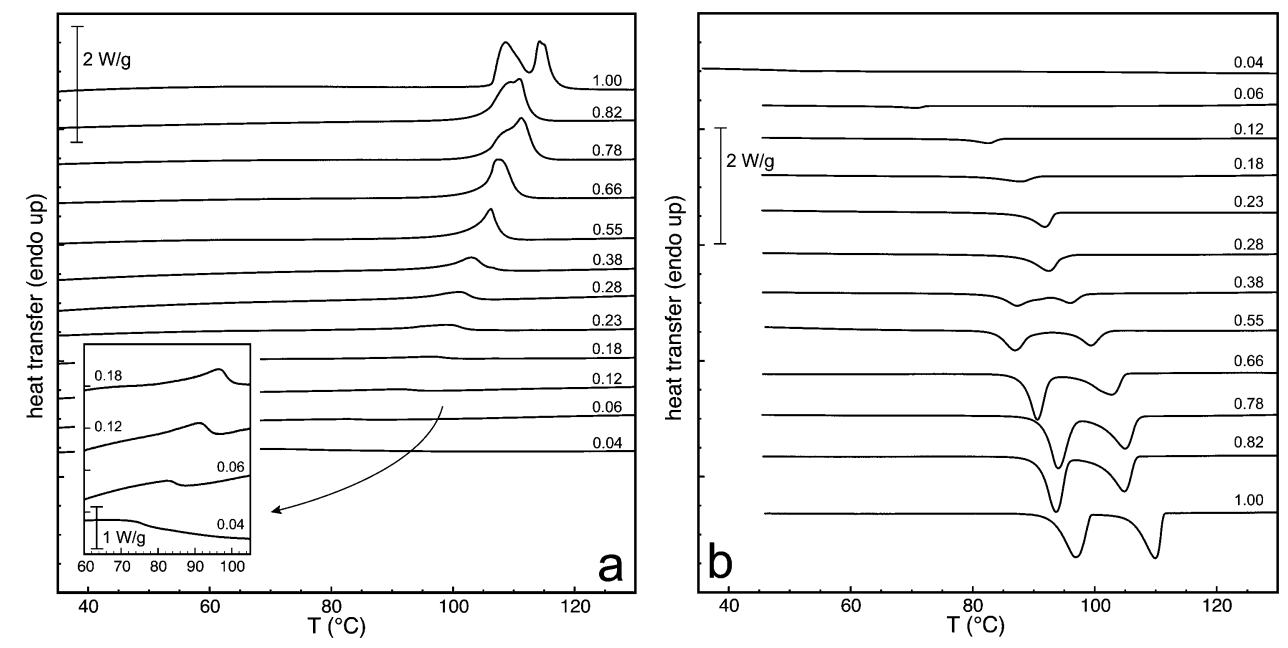

Fig. 3 (a) DSC first heating and (b) first cooling traces of $\mathrm{PbPa}$ in linseed oil ( $\left.2.5 \mathrm{~K} \mathrm{~min}^{-1}\right)$. Numbers indicate the molar fraction of PbPa. The inset shows a magnification of the DSC heating traces of low concentration samples. 


\subsection{ZnPa in linseed oil}

The endothermic and exothermic processes in mixtures of zinc palmitate and linseed oil (ZnPa-LO) are shown in Fig. 5. Though pure long-chain zinc soaps usually show a single melting transition, ${ }^{33}$ we occasionally observed additional peaks or peak asymmetry (e.g. mol fractions 0.22 and 0.75 in Fig. 5a), though these events were not reproducible. As before, peak areas were summed to yield a total transition enthalpy to allow comparison across the entire concentration range.

The melting and crystallization temperatures of the $\mathrm{ZnPa}-$ LO system are shown in Fig. 6. The melting temperature of ZnPa does not decrease as sharply at low concentrations as it does for both HPa and PbPa, suggesting an even lower solubility of $\mathrm{ZnPa}$ at room temperature than $\mathrm{PbPa}$. Indeed, using the fitted ideal solution line from eqn (1), we obtained a melting temperature and melting enthalpy very close to the reported values, ${ }^{33}$ and a solubility at $20{ }^{\circ} \mathrm{C}$ of roughly $4 \times 10^{-4} \mathrm{~mol} \%$ (Table 1 ).

The corresponding melting enthalpy shows a decreasing trend similar to $\mathrm{PbPa}$, dropping from $85.6 \pm 0.2 \mathrm{~kJ} \mathrm{~mol}^{-1}$ for pure $\mathrm{ZnPa}$ to just $11 \pm 2 \mathrm{~kJ} \mathrm{~mol}^{-1}$ at a $\mathrm{ZnPa}$ mol fraction of 0.05 . The relatively large deviation of the enthalpy values from the trend and the discrepancy between the experimental and fitted values of $\Delta^{\circ} H_{\text {melt }}$ are probably due to the inherent irreproducibility issues mentioned before.

A notable feature of the $\mathrm{ZnPa}-\mathrm{LO}$ system is the strong hysteresis between melting and crystallization temperatures. With values around $50{ }^{\circ} \mathrm{C}$ over the entire concentration range, supercooling is significantly larger than in the HPa-LO and PbPa-LO systems. Since pure ZnPa also shows strong hysteresis, the slow crystallization rate seems to be inherent to zinc soaps and it is not strongly influenced by the linseed oil medium. Interestingly, Barman and Vasudevan report that temperature hysteresis for zinc soaps becomes smaller with decreasing alkyl chain length, ${ }^{33}$ which suggests that molecular size or mobility is a contributing factor for the crystallization rate.

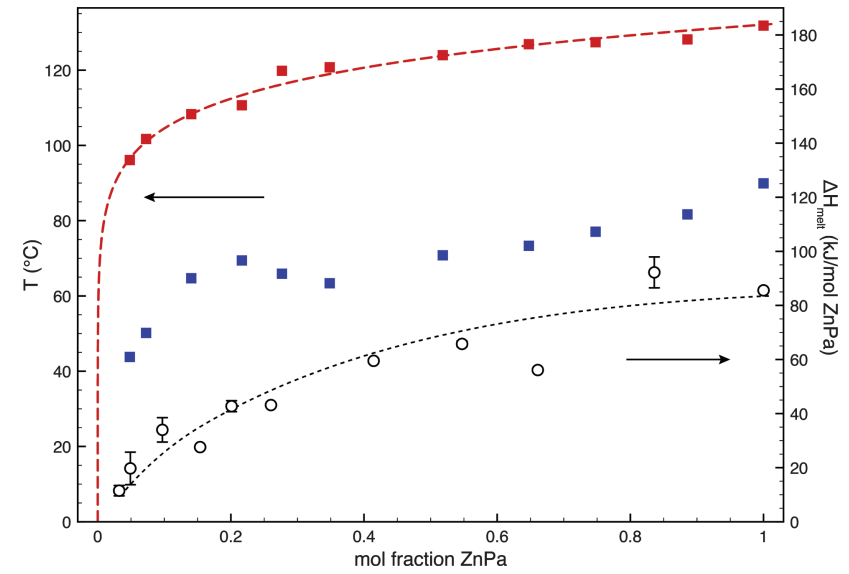

Fig. 6 Phase diagram of $\mathrm{ZnPa}$ in linseed oil with melting (red squares) and crystallization (blue squares) temperatures. The red dashed curve represents a fit of eqn (1). The corresponding melting enthalpy (circles) is indicated on the right axis (dotted curve added for clarity).

\subsection{Mixing enthalpy}

We found that the melting enthalpy of HPa in LO does not depend strongly on $\mathrm{HPa}$ concentration, while $\mathrm{PbPa}$ and $\mathrm{ZnPa}$ both show a decreasing melting enthalpy with decreasing concentration (Fig. 2, 4 and 6). Additionally, in all three cases the melting temperatures did not deviate strongly from ideal solution behavior. To further investigate these observations, we can divide the total measured enthalpy in each system as determined from DSC traces into a melting and a mixing process:

$$
\Delta H_{\text {total }}=\Delta H_{\text {melt }}+\Delta H_{\text {mix }} .
$$

Expressed per total number of molecules in the mixture, $\Delta H_{\text {total }}$ is linearly proportional to the concentration of $\mathrm{HPa}, \mathrm{PbPa}$ or $\mathrm{ZnPa}$ when $\Delta H_{\mathrm{mix}}=0$, i.e. in the case of ideal mixing and ideal solution behavior.
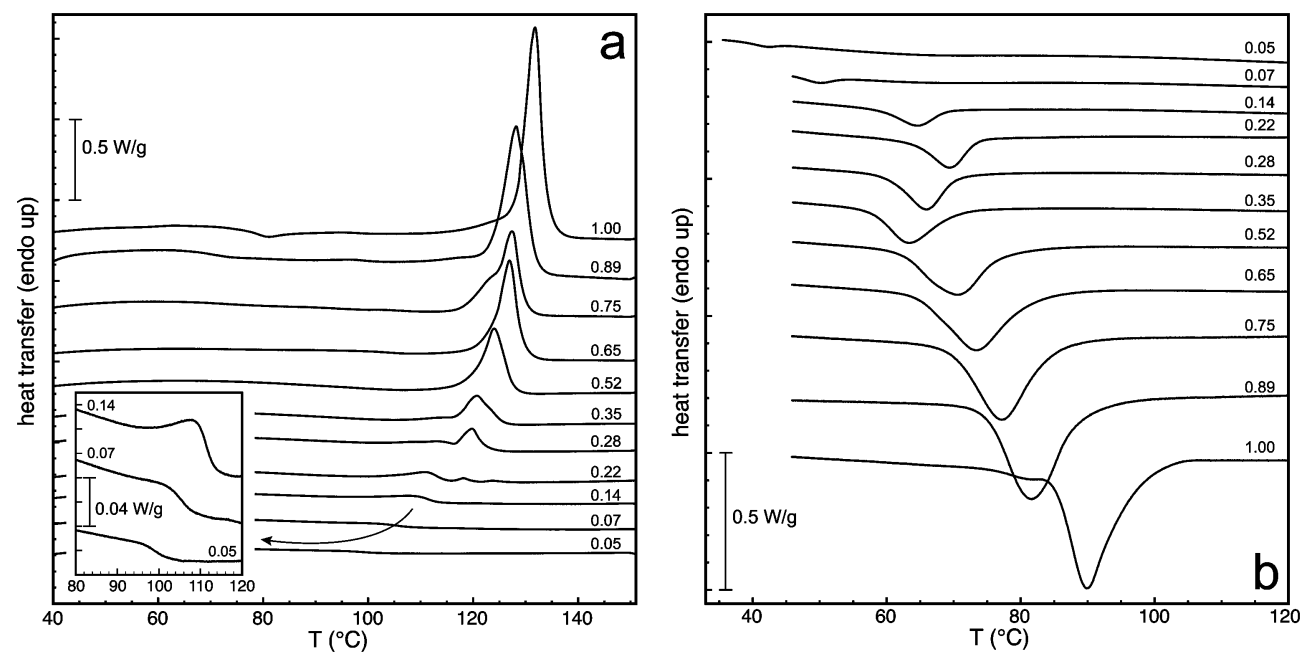

Fig. 5 DSC first heating (left) and first cooling (right) traces of $\mathrm{ZnPa}$ in linseed oil $\left(2.5 \mathrm{~K} \mathrm{~min}^{-1}\right)$. Numbers indicate the molar fraction of $\mathrm{ZnPa}$. The inset shows a magnification of the DSC heating traces of low concentration samples. 

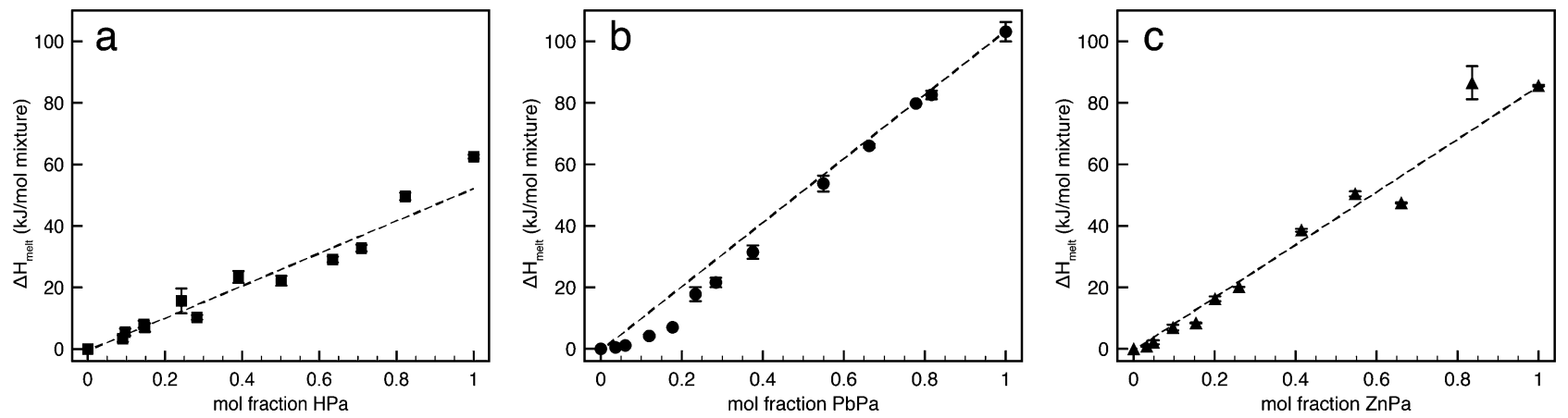

Fig. 7 The melting enthalpy of mixtures of (a) $\mathrm{HPa}$, (b) $\mathrm{PbPa}$ or (c) $\mathrm{ZnPa}$ and $\mathrm{LO}$ expressed as energy per total number of molecules in the mixture. Dashed lines represent ideal mixing, i.e. no interaction and a mixing enthalpy of 0 . Ideal mixing lines were based on the melting enthalpy of pure PbPa, ZnPa (both this work) and $\mathrm{HPa}$ (ref. 16).

Fig. 7 shows the measured total enthalpy change of the HPa$\mathrm{LO}, \mathrm{PbPa}-\mathrm{LO}$ and $\mathrm{ZnPa}-\mathrm{LO}$ systems upon heating as a function of concentration. In all three cases, the enthalpy values lie close to the ideal mixing line (i.e. $\Delta H_{\text {mix }}$ is small), which is in agreement with the excellent fits of ideal solution curves to the melting temperatures in the three investigated systems. For HPa-LO, the melting enthalpy values are spread on both sides of the ideal mixing line, while $\Delta H_{\text {mix }}$ seems to be tending towards small negative (i.e. exothermic) values for the metal soap systems, especially for PbPa-LO. Though the effect is certainly not very pronounced, a small negative mixing enthalpy would indicate that the interaction between metal soap and linseed oil molecules is slightly more favorable than the interaction between metal soap molecules.

\subsection{The effect of LO polymerization on crystallization}

The previous experiments were all conducted with liquid, nonpolymerized linseed oil. One approach to study the effect of polymerization of the oil medium on metal soap crystallization is to heat $\mathrm{HPa}-\mathrm{LO}, \mathrm{PbPa}-\mathrm{LO}$ and $\mathrm{ZnPa}-\mathrm{LO}$ mixtures in air above the melting temperature of the solute - where the mixtures are completely homogeneous - and record DSC cooling and heating traces of these mixtures after different polymerization times. This way, the starting point of the system is an amorphous state in which the palmitic acid or metal soap is molecularly dispersed throughout the system, which is how we envision fatty acid or metal soap crystallization to start in oil paint systems.

The melting and crystallization temperatures as well as crystallization enthalpy of each system as a function of polymerization time are shown in Fig. 8. For all three systems, we see a decreasing trend in the melting temperature, and a crystallization temperature that decreases faster. This increasing supercooling effect indicates that the crystallization process is slowing down as linseed oil starts to polymerize. Simultaneously, there is a strong decrease in the crystallization enthalpy with polymerization time. The effect is less pronounced for $\mathrm{HPa}$, while in contrast $\Delta H_{\text {crys }}$ practically vanished at polymerization times over 60 minutes for ZnPa-LO. Similar trends were found for the melting enthalpy. It seems, therefore, that in addition to an increasing supercooling required for crystallization, the driving force for crystallization is also strongly diminished as linseed oil becomes more polymerized.
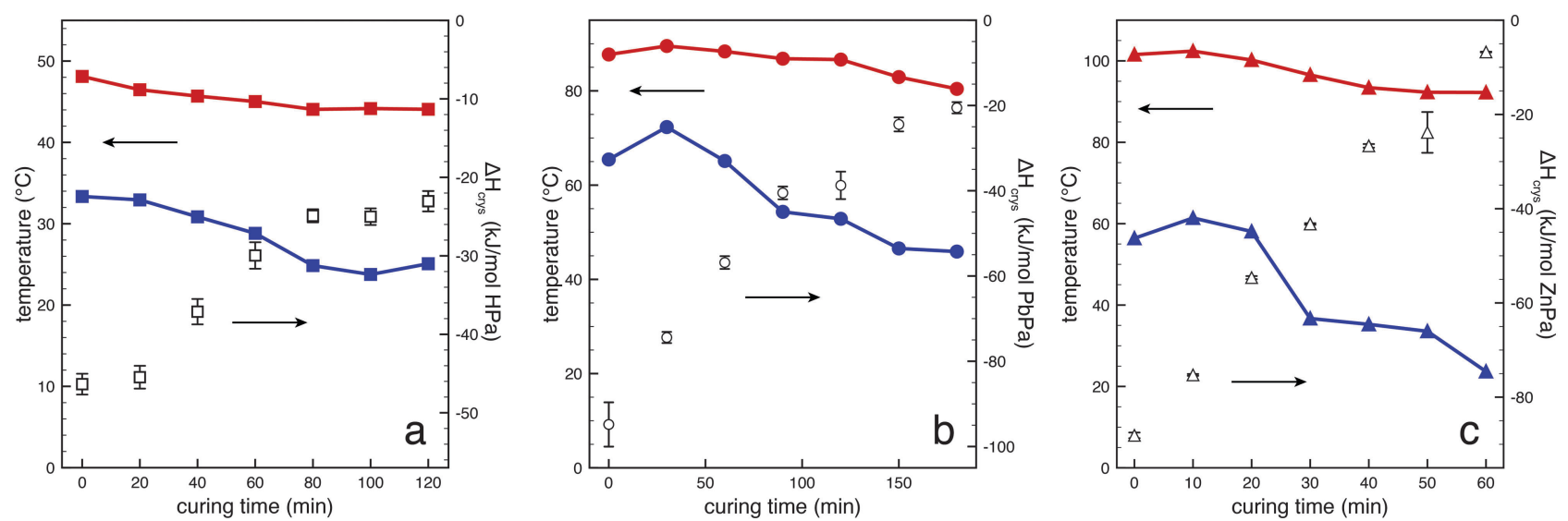

Fig. 8 Melting and crystallization temperatures (closed symbols) and crystallization enthalpy (open symbols) of (a) HPa-LO, (b) PbPa-LO and (c) $\mathrm{ZnPa}-\mathrm{LO}$ systems as a function of polymerization time in air at $150{ }^{\circ} \mathrm{C}$. Molar fractions are $0.40,0.11$ and 0.11 respectively. The heating rate is $10 \mathrm{~K} \mathrm{~min}^{-1}$, and the data represent the first heating and cooling runs. The lines connecting the points are added for clarity. 


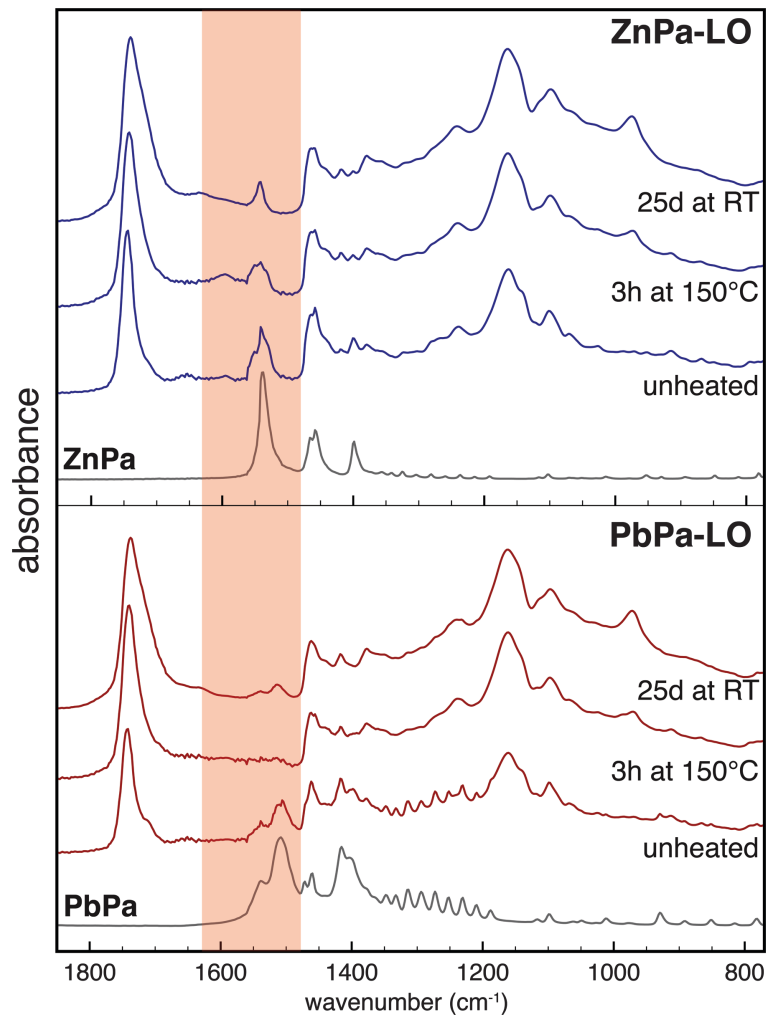

Fig. 9 Room temperature ATR-FTIR spectra of $\mathrm{PbPa}-\mathrm{LO}$ and $\mathrm{ZnPa}-\mathrm{LO}$ mixtures (both mol fraction of 0.11 metal soap) before heating, after heating at $150{ }^{\circ} \mathrm{C}$ for 3 hours, and after an additional 25 days under ambient conditions. Spectra of pure $\mathrm{PbPa}$ and $\mathrm{ZnPa}$ are included for comparison. The orange area indicates the spectral region associated with $\nu_{\mathrm{a}} \mathrm{COO}^{-}$vibrations. All spectra of mixtures were normalized to the $\mathrm{CH}_{2}$ band at $2922 \mathrm{~cm}^{-1}$.

The decreasing trend in crystallization enthalpy with the degree of LO polymerization for $\mathrm{PbPa}-\mathrm{LO}$ and $\mathrm{ZnPa}-\mathrm{LO}$ systems points to either stabilization of the initial amorphous state, destabilization of the crystalline end state, or a combination of these two effects. The states of the PbPa-LO and ZnPa-LO systems before and after partial polymerization were investigated by ATR-FTIR spectroscopy, as shown in Fig. 9. Before polymerization, $\mathrm{PbPa}$ in $\mathrm{LO}$ shows well-defined $\nu_{\mathrm{a}} \mathrm{COO}^{-}$bands at $1510 \mathrm{~cm}^{-1}$ and $1539 \mathrm{~cm}^{-1}$, as well as a complete series of $\mathrm{CH}_{2}$ progression bands in the $1200-1400 \mathrm{~cm}^{-1}$ region, which indicates all-trans alkyl chains and a crystalline metal soap structure. ${ }^{33}$ After polymerization by heat treatment at $150{ }^{\circ} \mathrm{C}$ for 3 hours, the progression bands are no longer visible in the spectrum at room temperature and the spectral region associated with $\nu_{\mathrm{a}} \mathrm{COO}^{-}$vibrations shows only weak ill-defined absorbance, suggesting that there is very little crystallinity left. Though endo- and exothermic events were still observed for this system (see Fig. 8b), the final state is evidently not the same as in non-polymerized systems. After an additional 25 days under ambient conditions, however, the signature $\nu_{\mathrm{a}} \mathrm{COO}^{-}$bands of $\mathrm{PbPa}$ have (partially) returned, which means that a relatively slow rearrangement process is at play that optimizes the coordination of the carboxylate groups around the lead ions. Such a slow crystallization process has been observed previously for mixtures of magnesium stearate and ethylene/acrylic acid ionomers. ${ }^{19}$

The behavior of the $\mathrm{ZnPa}-\mathrm{LO}$ system is less pronounced, as shown in Fig. 9. In general, the $\mathrm{CH}_{2}$ progression bands tend to be much weaker for $\mathrm{ZnPa}$ compared to $\mathrm{PbPa}$, which makes it difficult to observe them in diluted $\mathrm{ZnPa}-\mathrm{LO}$ systems. However, it can be seen that the characteristic $\nu_{\mathrm{a}} \mathrm{COO}^{-}$band of $\mathrm{ZnPa}$ at $1539 \mathrm{~cm}^{-1}$ narrows noticeably when the partially polymerized system is left for 25 days under ambient conditions, suggesting zinc coordination that becomes more defined over time.

It is important to note that an identical polymerization time in different systems does not need to correspond to an identical degree of oxidation or polymerization. It is known that various metal complexes or metal ions, including lead and zinc, have a strong effect on the rate of oxidative drying processes in linseed oil. ${ }^{29}$ Therefore, we measured ATR-FTIR spectra of HPa-LO, $\mathrm{PbPa}-\mathrm{LO}$ and $\mathrm{ZnPa}-\mathrm{LO}$ systems as a function of heating time at $150{ }^{\circ} \mathrm{C}$. As a measure of the degree of polymerization, the intensity of the $\mathrm{C}=\mathrm{CH}$ stretching band at $3010 \mathrm{~cm}^{-1}$ was plotted against heating time for all three systems, as shown in Fig. 10. The intensity of this band decreases during polymerization as the $\mathrm{C}=\mathrm{C}$ bonds are oxidized by molecular oxygen. ${ }^{36}$ In order to obtain quantitative data, the concentration of the ester $\mathrm{C}=\mathrm{O}$ bond was assumed to be constant and used as an internal standard (i.e. all spectra were normalized to the prominent $\mathrm{C}=\mathrm{O}$ stretch vibration band at $1741 \mathrm{~cm}^{-1}$ ). Fig. 10 reveals that the presence of $\mathrm{PbPa}$ or $\mathrm{ZnPa}$ does indeed accelerate the oxidation of linseed oil. However, the oxidation rate in the $\mathrm{ZnPa}-\mathrm{LO}$ system is only slightly higher than in PbPa-LO. The significantly faster decrease of the crystallization temperature and enthalpy with heating time compared to $\mathrm{PbPa}-\mathrm{LO}$ and HPa-LO that was observed for $\mathrm{ZnPa}-\mathrm{LO}$ in Fig. 8 is therefore a property of $\mathrm{ZnPa}$ itself, and not just caused by the higher degree of polymerization in the ZnPa-LO system. Apparently, then, the $\mathrm{ZnPa}$ crystallization process is much more sensitive to changes in the

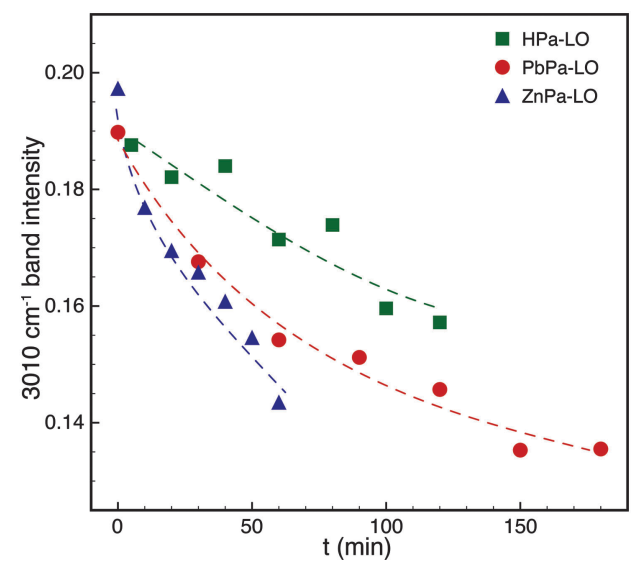

Fig. 10 Comparison of the intensity of the $\mathrm{C}=\mathrm{CH}$ band at $3010 \mathrm{~cm}^{-1}$ in the ATR-FTIR spectra of $\mathrm{HPa}-\mathrm{LO}, \mathrm{PbPa}-\mathrm{LO}$ and $\mathrm{ZnPa}-\mathrm{LO}$ systems as a function of curing time at $150{ }^{\circ} \mathrm{C}$ in air. The band intensity is reported relative to the $\mathrm{C}=\mathrm{O}$ band at $1741 \mathrm{~cm}^{-1}$ to correct for variations in the amount of sample in direct contact with the ATR crystal. Dashed curves are added for clarity. 
viscosity or chemical composition of the surrounding oil than either the PbPa or HPa crystallization process.

\section{Discussion}

ZnPa-LO stands out among the model systems we have investigated with differences between the melting and crystallization temperatures around $50{ }^{\circ} \mathrm{C}$, pointing to a slow crystallization process. We propose that differences in the molecular bilayer structure of $\mathrm{ZnPa}$ as compared to $\mathrm{PbPa}$ and $\mathrm{HPa}$ are the cause of this slower crystallization. Considering the crystalline structure of $\mathrm{ZnPa},{ }^{32,37}$ we observe that the alkyl chains in opposing planes are tilted with respect to each other, while they are completely aligned in lead soaps ${ }^{14,38}$ and palmitic acid. ${ }^{39}$ Moreover, the crystal plane containing the zinc ions in $\mathrm{ZnPa}$ is a polymeric 2D-network that is composed entirely of covalent $\mathrm{Zn}-\mathrm{OCO}-\mathrm{Zn}$ bonds, while crystalline $\mathrm{PbPa}$ forms covalent polymer strings in only one dimension and crystalline palmitic acid consists of stacked HPa dimers (see Fig. S2 to S4 in the ESI $\dagger$ for illustrations of these molecular structures).

Additional evidence for significant molecular changes during ZnPa crystallization was found by comparing the FTIR spectra of molten and crystalline metal soaps. In Fig. S5 (ESI $\dagger$ ), we see that the $\nu_{\mathrm{a}} \mathrm{COO}^{-}$band in $\mathrm{ZnPa}$ is split into three new bands upon melting, while the $\nu_{\mathrm{a}} \mathrm{COO}^{-}$band in $\mathrm{PbPa}$ merely broadens slightly. ${ }^{31}$ This contrast suggests that a much larger structural reordering is necessary around the carboxylate groups for $\mathrm{ZnPa}$ during the crystallization process than for Pba. These differences in structural reordering that occur upon melting of $\mathrm{ZnPa}$ on the one hand and $\mathrm{PbPa}$ and $\mathrm{HPa}$ on the other might result in a lower probability of forming a suitable nucleus for $\mathrm{ZnPa}$ crystallization (i.e. a higher energy barrier), or a slower rate of crystal growth from dissolved molecular species.

The DSC measurements of palmitic acid, lead palmitate and zinc palmitate in linseed oil reported above have important implications for our understanding of metal soap degradation phenomena in oil paintings. For liquid linseed oil, we showed that $\mathrm{HPa}, \mathrm{PbPa}$ and $\mathrm{ZnPa}$ all exhibit near-ideal solution behavior, allowing us to determine with sufficient confidence that the solubility of the metal soaps at room temperature is very low. The finding that HPa is orders of magnitude more soluble in linseed oil than the metal soaps suggests that the medium will not reach the saturation concentration of HPa (or other 'free' fatty acids) as long as there are metal ions available to form a metal soap complex. In other words, the formation of crystalline fatty acid efflorescence in paintings can only occur when metal ions are absent in the binding medium, for example in paint systems that contain only organic pigments or inorganic pigments that hardly leach any metal ions into the oil binding medium.

In our polymerized systems, the melting temperature of $\mathrm{PbPa}$ and $\mathrm{ZnPa}$ dropped slightly, but we can expect that the solubility of these compounds in cured linseed oil films remains low. Therefore, once metal ions and fatty acids form their metalcarboxylate bonds in a paint film, this system is inherently unstable, and the metal soaps will crystallize spontaneously. However, whether metal soap crystallization does actually occur is a question of kinetics. In our experiments, the supercooling needed for crystallization of both $\mathrm{PbPa}$ and $\mathrm{ZnPa}$ increased significantly with the degree of polymerization, and we may expect the crystallization temperature to become even lower for fully cross-linked or aged oil paint systems. Especially for ZnPa, the temperature hysteresis becomes so large that the crystallization temperature could drop below room temperature. If this is the case, it would mean that the probability of forming a critical nucleus for crystallization is very low for zinc soaps in dry oil paint films. When zinc soap complexes are formed in those systems, it may take a very long time before any crystallization occurs, if at all. In this scenario, the paint medium might become kinetically trapped in a state of amorphous zinc soaps.

Given a situation in which 'free' saturated fatty acids exist in a polymerized oil paint system and assuming that metal soaps are at best sparingly soluble in those systems, any factors that increase the probability of forming critical crystallization nuclei will drastically increase the chance that a painting develops damaging metal soap related degradation phenomena. As long as the extent of pigment loss due to metal soap formation does not lead to visible color changes and the metal soaps remain amorphous, damage to the appearance or structural integrity of the paint is rather limited. Factors that may increase the nucleation rate include the existence of (micro-)cracks in the paint or the exposure of the paint film to solvents that allow metal soap units to rearrange at a quicker rate into energetically favorable conformations than they could achieve in a dry polymer film.

It is of particular interest in the light of our findings to consider the technique of wax-resin lining of oil paintings. Until the mid-1970s, it was common conservation practice to attach a new stretch of canvas as a reinforcement to the back of a weakened canvas painting with a combination of wax and natural resin. ${ }^{40}$ During the lining process, heat and pressure were applied to melt the wax and ensure a good bonding between the old and new canvas, or even to consolidate unstable paints. Even though the temperatures involved were quite moderate, about $50-75{ }^{\circ} \mathrm{C}$, such short heating periods may have contributed to the issue of metal soap aggregates in oil paintings. Besides simply enhancing the mobility of all molecules and metal ions in the polymerized binding medium, elevated temperatures strongly increase the concentration of dissolved saturated fatty acids and metal soap species. The combination of these two effects helps the precipitation of crystalline metal soaps once the paint cools back down to room temperature. Whether the contribution of this increased crystallization rate is significant in relation to the timespan that the painting spends at room temperature remains to be investigated. However, this example certainly shows that consideration of the phase behavior of metal soaps and their crystallization kinetics can assist in understanding how conservation treatments or environmental conditions affect the state of an oil painting. 


\section{Conclusions}

At room temperature, HPa has a solubility of approximately $6.6 \mathrm{~mol} \%$ ( $87 \mathrm{mM})$ in linseed oil. In contrast, lead and zinc soaps are practically insoluble in liquid linseed oil, and this insolubility is not significantly affected by the polymerization of the oil medium. The excellent fit of the relation describing ideal solubility to the melting temperatures in HPa-LO, PbPa-LO and $\mathrm{ZnPa}-\mathrm{LO}$ mixtures combined with the small or nonexistent contribution of a mixing enthalpy to the total melting enthalpy change both indicate that there is very limited favorable interaction between either $\mathrm{HPa}, \mathrm{PbPa}$ or $\mathrm{ZnPa}$ and linseed oil. Therefore, we can conclude that the crystallization of metal soaps in oil paint layers is a spontaneous and irreversible phenomenon that is caused simply by the very low solubility of metal soaps in oil paints.

This conclusion has important implications for the conservation practice of oil paintings. The wide variation in the degree of metal soap crystallization and aggregation in oil paintings across the world must be caused by differences in the concentrations of the reactants that form metal soaps in the paint layers (i.e. metal ions and 'free' fatty acids), or by differences in the conditions that affect the kinetics of metal soap crystallization. In the last category, the presence of microscopic fractures in the paint, the exposure to solvents during cleaning or the application of heat may all play an important role. Differences in the relative rate of nucleation and growth of metal soap crystalline phases could also explain why there is such a great variety in the morphology of metal soaps found in oil paint layers. Future research efforts will be directed towards unravelling the oil paint properties and environmental factors that influence the nucleation and growth kinetics during metal soap crystallization, in order to compile a comprehensive explanation of metal soap-related degradation processes in oil paints.

\section{Acknowledgements}

The authors wish to thank Sanne Berbers for performing some of the DSC experiments, and Francisco Martínez Casado for useful discussions on the structure of metal soaps. This work is part of the PAinT project, supported by the Science4Arts program of the Dutch Organization for Scientific Research (NWO).

\section{References}

1 C. Higgit, M. Spring and D. Saunders, National Gallery Technical Bulletin, 2003, 24, 75-95.

2 P. Noble, A. van Loon and J. Boon, ICOM Committee for Conservation, 14th Triennial Conference Preprints, The Hague, 12-16 September 2005, London, 2005, pp. 496-503.

3 K. Keune and J. Boon, Stud. Conserv., 2007, 52, 161-176.

4 K. Keune, A. van Loon and J. J. Boon, Microsc. Microanal., 2011, 17, 696-701.

5 G. Osmond, J. J. Boon, L. Puskar and J. Drennan, Appl. Spectrosc., 2012, 66, 1136-1144.
6 K. Keune and G. Boevé-Jones, Issues in Contemporary Oil Paint, 2014, pp. 283-294.

7 G. Osmond, B. Ebert and J. Drennan, AICCM Bulletin, 2014, 34, 4-14.

8 R. Williams, Proceedings of the 14th Annual IIC-CG Conference, May 27-30, 1988, Toronto, 1988, pp. 65-84.

9 E. Ordonez and J. Twilley, Anal. Chem., 1997, 69, 416A-422A. $10 \mathrm{~J}$. van der Berg, PhD thesis, University of Amsterdam, 2002.

11 M. S. Akanni, E. K. Okoh, H. D. Burrows and H. A. Ellis, Thermochim. Acta, 1992, 208, 1-41.

12 A. Sanchez Arenas, M. V. Garcia, M. I. Redondo, J. A. R. Cheda, M. V. Roux and C. Turrion, Liq. Cryst., 1995, 18, 431-441.

13 O. Berkesi, T. Katona, I. Dreveni, J. Andor and J. Mink, Vib. Spectrosc., 1995, 8, 167-174.

14 F. Martínez Casado, M. García Pérez, M. Redondo Yélamos, J. Rodríguez Cheda, A. Sánchez Arenas, S. López-Andrés, J. Garca-Barriocanal, A. Rivera, C. León and J. Santamaría, J. Phys. Chem. C, 2007, 111, 6826-6831.

15 F. J. Martínez-Casado, M. Ramos-Riesco, J. A. RodríguezCheda, F. Cucinotta, A. Fernández-Martínez, L. Garrido, E. Matesanz and L. Marchese, J. Mater. Chem. C, 2014, 2, 9489-9496.

16 F. O. Cedeño, M. M. Prieto, A. Espina and J. R. García, Thermochim. Acta, 2001, 369, 39-50.

17 R. Weiss, J. Appl. Polym. Sci., 1983, 28, 3321-3332.

18 P. Antony and S. De, Polymer, 1999, 40, 1487-1493.

19 K. Wakabayashi and R. A. Register, Polymer, 2006, 47, 2874-2883.

20 P. Musto, D. Larobina, S. Cotugno, P. Straffi, G. Di Florio and G. Mensitieri, Polymer, 2013, 54, 685-693.

21 Y. Ikeda, Y. Yasuda, T. Ohashi, H. Yokohama, S. Minoda, H. Kobayashi and T. Honma, Macromolecules, 2015, 48, 462-475.

22 R. Grayburn, M. Dowsett, M. de Keersmaecker, E. Westenbrink, J. A. Covington, J. B. Crawford, M. Hand, D. Walker, P. A. Thomas, D. Banerjee and A. Adriaens, Corros. Sci., 2014, 82, 280-289.

23 M. De Keersmaecker, O. V. D. Berg, K. Verbeken, D. Depla and A. Adriaens, J. Electrochem. Soc., 2015, 162, C167-C179.

24 A. Mesbah, S. Jacques, E. Rocca, M. François and J. Steinmetz, Eur. J. Inorg. Chem., 2011, 1315-1321.

25 S. Mishra, S. Daniele and L. G. Hubert-Pfalzgraf, Chem. Soc. Rev., 2007, 36, 1770.

26 J. J. Hermans, K. Keune, A. van Loon and P. D. Iedema, J. Anal. At. Spectrom., 2015, 30, 1600-1608.

27 R. L. Feller, Accelerated aging: photochemical and thermal aspects, The Getty Conservation Institute, Los Angeles, 1994.

28 J. D. J. van den Berg, N. D. Vermist, L. Carlyle, M. Holčapek and J. J. Boon, J. Sep. Sci., 2004, 27, 181-199.

29 R. van Gorkum and E. Bouwman, Coord. Chem. Rev., 2005, 249, 1709-1728.

30 M. Cotte, E. Checroun, J. Susini and P. Walter, Appl. Phys. A: Mater. Sci. Process., 2007, 89, 841-848.

31 T. Ishioka, K. Maeda, I. Watanabe, S. Kawauchi and M. Harada, Spectrochim. Acta, Part A, 2000, 56, 1731-1737. 
32 J. J. Hermans, K. Keune, A. van Loon, R. W. Corkery and P. D. Iedema, Polyhedron, 2014, 81, 335-340.

33 S. Barman and S. Vasudevan, J. Phys. Chem. B, 2006, 110, 22407-22414.

34 P. Atkins and J. de Paula, Atkins' Physical Chemistry, Oxford University Press, Oxford, 2002, 7th edn, pp. 177-178.

35 C. Himawan, V. M. Starov and A. G. F. Stapley, Adv. Colloid Interface Sci., 2006, 122, 3-33.

36 J. Mallégol, J. Lemaire and J.-L. Gardette, Prog. Org. Coat., 2000, 39, 107-113.
37 F. Lacouture, J. Peultier, M. François and J. Steinmetz, Acta Crystallogr., Sect. C: Cryst. Struct. Commun., 2000, 56, 556-557.

38 F. Lacouture, M. François, C. Didierjean, J.-P. Rivera, E. Rocca and J. Steinmetz, Acta Crystallogr., Sect. C: Cryst. Struct. Commun., 2001, 57, 530-531.

39 E. Moreno, R. Cordobilla, T. Calvet, F. J. Lahoz and A. I. Balana, Acta Crystallogr., Sect. C: Cryst. Struct. Commun., 2006, 62, 0129-0131.

40 S. Hackney, J. Reifsnyder, M. te Marvelde and M. Scharff, in Conservation of Easel Paintings, ed. J. Hill Stoner and R. Rushfield, Routledge, London, 2012, pp. 415-452. 